\title{
Evaluation of atrial volume and function with magnetic resonance imaging in hypoplastic left heart
}

Chodchanok Vijarnsorn ${ }^{1 *}$, Kimberley A Myers², David J Patton², Cinzia Crawley ${ }^{1}$, Michelle Noga ${ }^{1}$, Edythe B Tham ${ }^{1}$

From 16th Annual SCMR Scientific Sessions

San Francisco, CA, USA. 31 January - 3 February 2013

\section{Background}

Increased left atrial volume and reduced atrial emptying are predictors of adverse cardiac events in adults. The atrium in single ventricle hearts contributes to ventricular filling to a greater degree than in normal hearts.

Standardized methods of determining atrial function in single ventricles are lacking. We aimed to assess the feasibility and reproducibility of quantifying atrial volumes and function in hypoplastic left heart (HLH) prior to the Glenn operation.
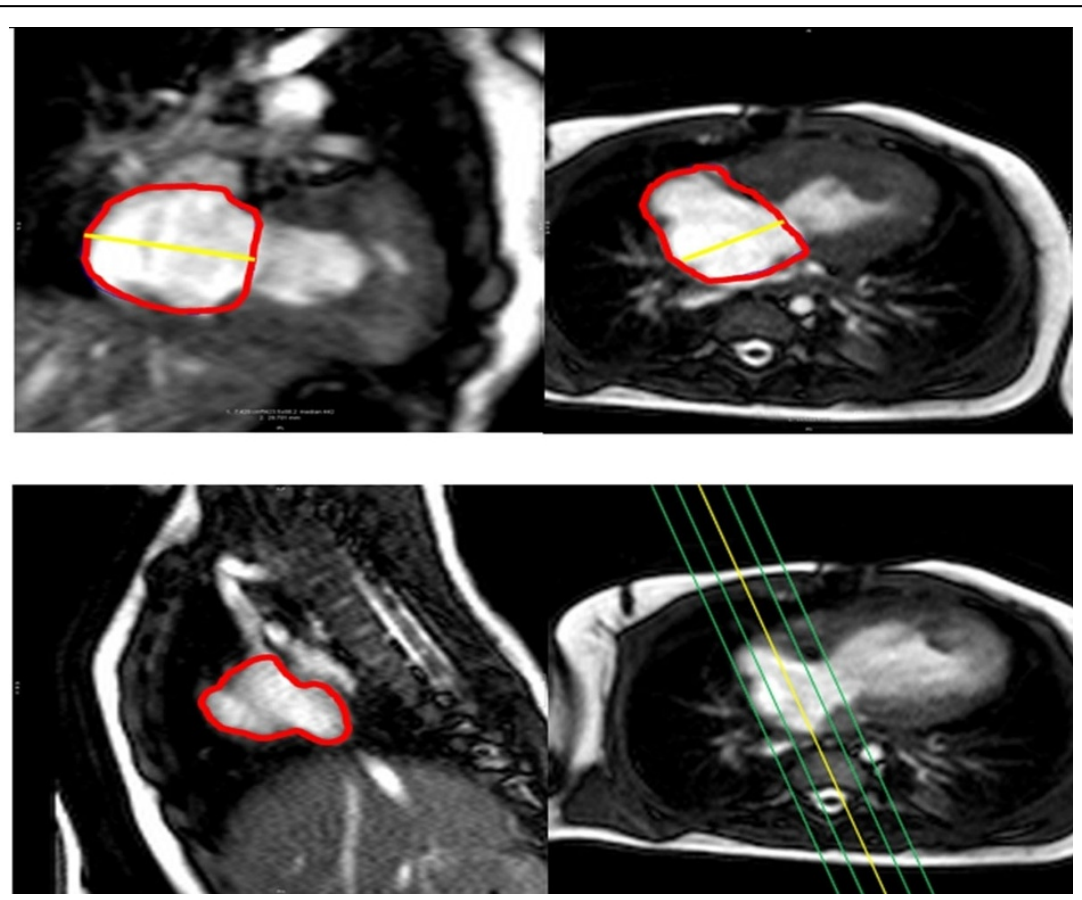

Figure 1 Measurement of right atrial volumes in hypoplastic left heart in $4 \mathrm{CH}, 2 \mathrm{CH}$ and $\mathrm{SAO}$ stack.

Mazankowski Alberta Heart Institute, University of Alberta, Edmonton, $A B$,

Canada

Full list of author information is available at the end of the article 
Table 1 Comparison of aEDV, aESV and aEF between 3D-SAO and monoplane 4CH, 2CH and biplane method ( $\mathrm{n}=14$ )

\begin{tabular}{|c|c|c|c|c|c|}
\hline & Mean & $\begin{array}{l}\text { Mean differences (with 3D } \\
\text { SAO) }\end{array}$ & $\mathrm{p}$-value & $\begin{array}{l}\text { Correlation } \\
\text { (R) }\end{array}$ & $\mathrm{p}$-value \\
\hline $\begin{array}{c}\mathrm{aEDV}(\mathrm{mL})-3 \mathrm{D} \mathrm{SAO}-4 \mathrm{CH}- \\
2 \mathrm{CH} \text { - biplane }\end{array}$ & $\begin{array}{c}15.77 \pm 3.0215 .55 \pm 4.0613 .68 \pm \\
2.9914 .82 \pm 3.02 \\
\end{array}$ & $\begin{array}{c}--0.22 \pm 3.01-2.08 \pm 1.61- \\
0.95 \pm 1.67\end{array}$ & $\begin{array}{c}-0.78<0.001 \\
0.05 \\
\end{array}$ & $\begin{array}{c}-0.680 .84 \\
0.84 \\
\end{array}$ & $\begin{array}{c}-0.008<0.001 \\
<0.001\end{array}$ \\
\hline $\begin{array}{l}\text { aESV }(\mathrm{mL}) \text { - 3D SAO - 4CH - } \\
\text { 2CH - biplane }\end{array}$ & $\begin{aligned} 8.73 \pm 2.287 .88 & \pm 2.617 .37 \pm 2.44 \\
7.71 & \pm 2.37\end{aligned}$ & $\begin{array}{c}--0.85 \pm 1.56-1.35 \pm 1.34 \\
-1.01 \pm 0.95 \\
\end{array}$ & $\begin{array}{c}-0.070 .002 \\
0.001 \\
\end{array}$ & $\begin{array}{c}-0.800 .84 \\
0.91 \\
\end{array}$ & $\begin{array}{c}-0.001<0.001 \\
<0.001 \\
\end{array}$ \\
\hline $\begin{array}{c}\mathrm{aEF}-3 \mathrm{D} \text { SAO }-4 \mathrm{CH}-2 \mathrm{CH}- \\
\text { biplane }\end{array}$ & $\begin{array}{c}0.45 \pm 0.080 .51 \pm 0.110 .47 \pm 0.09 \\
0.48 \pm 0.08\end{array}$ & $\begin{array}{c}-0.06 \pm 0.060 .02 \pm 0.040 .03 \\
\pm 0.03\end{array}$ & $\begin{array}{l}-0.0060 .07 \\
0.004\end{array}$ & $\begin{array}{c}-0.760 .88 \\
0.91\end{array}$ & $\begin{array}{c}-0.001<0.001 \\
<0.001\end{array}$ \\
\hline
\end{tabular}

Values are expressed by mean \pm SD Statistical significance at $p$ value $<0.05$

\section{Methods}

Fourteen patients with HLH prior to Glenn surgery (4.2 \pm 1.2 months) underwent CMR cine imaging in 2 chamber $(2 \mathrm{CH})$ plane, 4 chamber $(4 \mathrm{CH})$ plane and $3 \mathrm{D}$-atrial shortaxial oblique (3D-SAO) stack (Figure 1). Right atrial volumes and atrial ejection fraction $(\mathrm{aEF})$ were assessed by 3 methods: 1) biplane $=0.85 \mathrm{x}$ area $4 \mathrm{CH} \mathrm{x}$ area2 $\mathrm{CH} /$ (length4CH + length2CH)/2; 2) $2 \mathrm{CH}$ monoplane; and 3) $4 \mathrm{CH}$ monoplane area-length formula: $=0.85 x$ area2/length in end-diastole (aEDV) and end-systole (aESV). These 3 methods were compared to $3 \mathrm{D}-\mathrm{SAO}$ volume and aEF by paired t-test. Agreement was compared using mean differences (diff) and Spearman correlation. Interobserver reproducibility was analyzed by intra class correlation (ICC). Correlation of aEDV, aESV and aEF with RV mass was analyzed using Spearman correlation.

\section{Results}

Monoplane 4CH and biplane methods showed high correlations for aEDV and aESV as well as small mean differences compared to 3D-SAO (Table 1). Despite a good correlation with $3 \mathrm{D}-\mathrm{SAO}$, the monoplane $2 \mathrm{CH}$ method underestimated aEDV and aESV. The aEF by all methods showed high correlations, but overestimated aEF when compared to 3D-SAO. Inter and intra observer variability demonstrated good agreement in both $3 \mathrm{D}$ SAO (aEDV: ICC 0.79, aESV:ICC 0.84, aEF 0.87; all $\mathrm{p}<0.001)$ and 4CH (EDV: ICC 0.73; p 0.001, ESV:ICC 0.68; $\mathrm{p}<0.001$, aEF 0.50, all $\mathrm{p}<0.02$ ). Increased RV mass index was related to increased atrial ESV index ( $\mathrm{R}-0.67$, $\mathrm{p} 0.03)$ and decreased aEF ( $\mathrm{R}-0.76, \mathrm{p} 0.01)$.

\section{Conclusions}

CMR atrial volume assessment is feasible in HLH prior to Glenn operation. The monoplane $4 \mathrm{CH}$ and biplane methods show the best correlate of atrial volumes and function. Increased RV mass may be associated with reduced atrial function.

\section{Funding}

None.

\section{Author details}

'Mazankowski Alberta Heart Institute, University of Alberta, Edmonton, $A B$, Canada. ${ }^{2}$ Alberta Children's Hospital, Calgary, AB, Canada.

Published: 30 January 2013

doi:10.1186/1532-429X-15-S1-041

Cite this article as: Vijarnsorn et al.: Evaluation of atrial volume and function with magnetic resonance imaging in hypoplastic left heart. Journal of Cardiovascular Magnetic Resonance 2013 15(Suppl 1):041.
Submit your next manuscript to BioMed Central and take full advantage of:

- Convenient online submission

- Thorough peer review

- No space constraints or color figure charges

- Immediate publication on acceptance

- Inclusion in PubMed, CAS, Scopus and Google Scholar

- Research which is freely available for redistribution

Submit your manuscript at www.biomedcentral.com/submit
C Biomed Central 